\title{
Influência da televisão no consumo alimentar e na obesidade em crianças e adolescentes: uma revisão sistemática
}

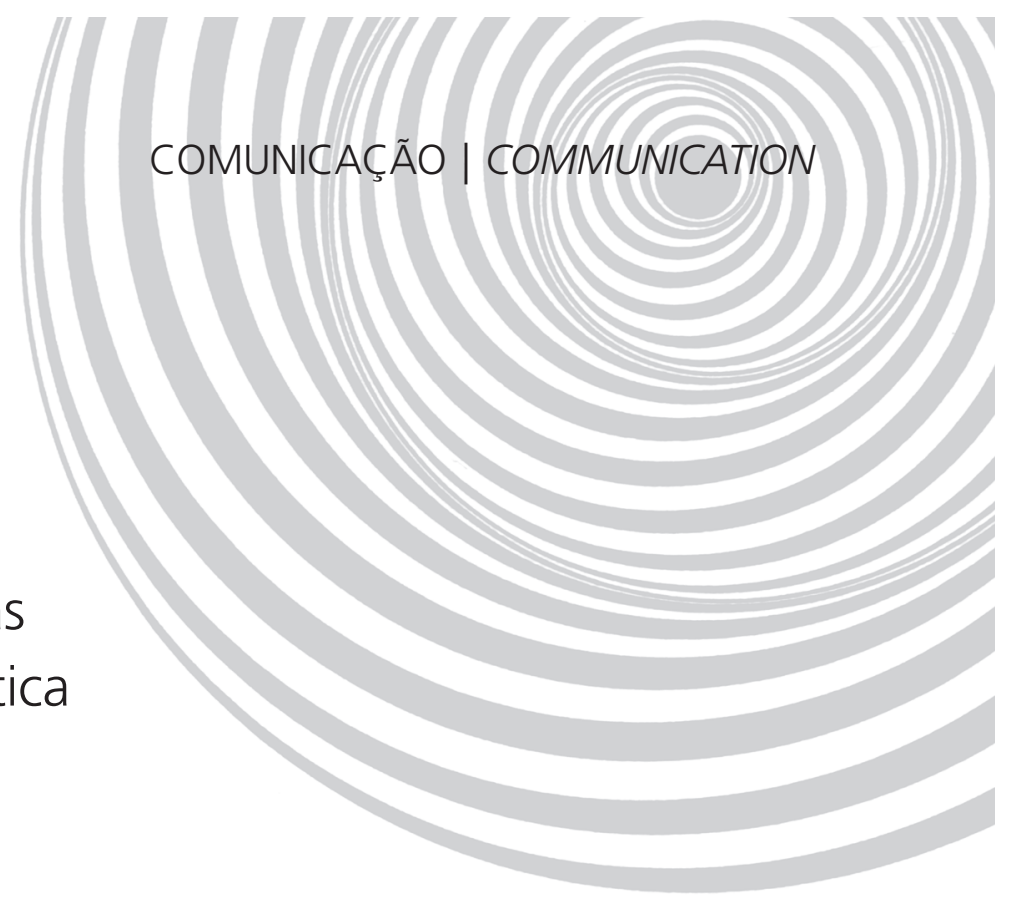

\author{
Television influence on food intake and \\ obesity in children and adolescents:
}

a systematic review

Camila Elizandra ROSSI ${ }^{1}$

Denise Ovenhausen ALBERNAZ1

Francisco de Assis Guedes de VASCONCELOS'

Maria Alice Altenburg de ASSIS 1

Patrícia Faria Di PIETRO'

RE S U M O

O objetivo deste trabalho foi identificar a influência da televisão no consumo alimentar e na obesidade em crianças e adolescentes. Realizou-se revisão sistemática nas bases MedLine, PubMed, SciELO-Brasil e Lilacs, capturando-se 73 artigos publicados entre 1997 e 2007. Definiu-se não analisar artigos com adultos ou pré-escolares, notas, comunicações, revisões, amostras de população específica (indígenas, por exemplo) e enferma (exceto obesidade), e estudos de intervenção, selecionando-se vinte artigos para análise. Em seis, dentre oito estudos brasileiros, observou-se associação significativa entre televisão e obesidade, e, em um destes, também se verificou associação positiva entre televisão e consumo alimentar. Nas investigações internacionais, observaram-se seis, dentre dez artigos, evidenciando associação entre televisão e obesidade, além de se identificar associação inversa entre televisão e tempo de atividade física. Em cinco artigos, do total de seis internacionais que investigaram o consumo alimentar, observou-se associação significativa entre televisão e ingestão alimentar, verificando-se que crianças e adolescentes que despendem maior tempo com a televisão tendem a ingerir menos frutas e verduras, e mais porções de salgadinhos, doces e bebidas com elevado teor de açúcar. A associação entre televisão e consumo alimentar foi evidente (85\% dos artigos), e a associação com obesidade apareceu em $60 \%$ dos artigos. Ao identificar-se que o tempo em frente à TV associa-se à inadequação dos hábitos alimentares e à redução da atividade física, revela-se o hábito de assistir à televisão como um importante fator que pode propiciar a obesidade entre crianças e adolescentes.

Termos de indexação: Adolescente. Consumo alimentar. Criança. Obesidade. Sobrepeso. Televisão.

\footnotetext{
1 Universidade Federal de Santa Catarina, Centro de Ciências da Saúde, Departamento de Nutrição, Pós-Graduação em Nutrição. Caixa Postal 476, Trindade, 88010-970, Florianópolis, SC, Brasil. Correspondência para/Correspondence to: C.E. ROSSI. E-mail: <camilarnutri@yahoo.com.br>.
} 


\section{A B S T R A C T}

The objective of this study was to identify the influence that watching television has on food intake and obesity in children and adolescents. A systematic review of the MedLine, PubMed, SciELO-Brasil and Lilacs databases was done which resulted in 73 articles published between 1997 and 2007. Articles with adults or preschoolers, notes, communications, reviews, samples of specific populations (Indians for example) or diseases (except for obesity) and intervention studies were excluded, resulting in 20 articles for analysis. In six of eight Brazilian studies, there was a significant association between watching television and obesity. In one of them, there was also a significant association between watching television and food intake. In six of ten international studies, there was a positive association between watching television and obesity and a negative association between times spent watching television and doing physical activity. In five out of six international studies that investigated food intake, there was a significant association between watching television and food intake. It was also found that children and adolescents who spend more time watching television tend to consume less fruits and vegetables and more salty snacks, sweets and sugar-rich beverages. The association between watching television and food intake was evident, found in $85 \%$ of the articles, and the association between watching television and obesity was found in $60 \%$ of the articles. Since time spent watching television is associated with unhealthy food habits and reduced levels of physical activity, it becomes an important factor in the promotion of obesity in children and adolescents.

Indexing terms: Adolescent. Food consummation. Child obesity. Overweight. Television.

\section{N T R O D U ÇÃ O}

Entre crianças e adolescentes, altas prevalências de obesidade têm sido relatadas em diversos países do mundo'. Na Europa ocorreu um aumento de dez vezes na taxa de obesidade nestas faixas etárias, desde a década de 1970 até a atualidade 2 . Um estudo sobre a tendência de sobrepeso, obesidade e baixo peso das crianças e adolescentes nos Estados Unidos da América (EUA), Brasil, China e Rússia, mostrou um aumento da incidência de sobrepeso no Brasil (4,1 para $13,9 \%)$, na China (6,4 para $7,7 \%)$ e nos EUA $(15,4$ para $25,6 \%)^{3}$.

Dentre os motivos para esse aumento podem-se citar os fatores comportamentais, tais como o estilo de vida sedentário e o excessivo consumo de alimentos de alta densidade energética ${ }^{4-6}$. Nesse contexto, acredita-se que o tempo excessivo dedicado a assistir à Televisão (TV) possa ser um marcador para identificação de baixos níveis de atividade física e também de práticas alimentares pouco saudáveis.

Em relação ao sedentarismo, Pimenta \& Palma ${ }^{7}$ observaram que a média de tempo despendido em frente à TV (2,6 horas/dia) era maior do que a média de tempo despendido com atividade física (1,1 hora/dia), entre escolares de dez e onze anos, matriculados em uma escola no município do Rio de Janeiro. Em Florianópolis, um estudo realizado em 2002 revelou que crianças entre sete e nove anos de idade despendiam, em média, 3,3 horas/dia em frente à TV, sendo que somente $35,7 \%$ dentre 1689 crianças realizavam algum tipo de esporte, além daquele praticado em horário escolar8.

Quanto às práticas alimentares, Salmon et al..$^{9}$ e Almeida et al. ${ }^{10}$ observaram associação entre o baixo consumo de frutas e hortaliças e a elevada audiência de TV, entre adolescentes. Almeida et $a l .^{10}$, ao analisarem a TV brasileira, observaram que $27,4 \%$ das propagandas referiam-se a alimentos, e que a veiculação desses comerciais distribuía-se por todos os períodos do dia. Na TV dos EUA, Story \& Faulkner ${ }^{11}$ identificaram que $60,0 \%$ dos comerciais consistiam em publicidade de refrigerantes e outros produtos açucarados. Outro estudo, realizado com escolares de $3^{\text {as }}$ e $5^{\text {as }}$ séries residentes em uma cidade dos EUA, mostrou que $16,6 \%$ e $26,2 \%$ da energia média consumida por dia era ingerida enquanto se assistia à TV, em dias de semana e de final de semana, respectivamente ${ }^{12}$. Em Florianópolis, Fiates et al. ${ }^{13}$ observaram que crianças de sete a dez anos de idade assistiam à TV diariamente, em especial durante as refeições, consumindo 
desde balas a refeições inteiras, em qualquer ambiente da residência onde estivesse a TV (cozinha, sala ou quarto). Apesar disso, as crianças relataram existir alguma forma de interferência de seus pais nas escolhas alimentares, mas não no tempo em frente à TV.

O objetivo deste artigo é verificar se o tempo despendido em frente à televisão exerce influência no consumo alimentar e na obesidade em crianças e adolescentes, tendo como base empírica de análise revisão sistemática de artigos científicos publicados entre 1998 a 2006.

\section{MÉ T O D O S}

Realizou-se revisão sistemática de artigos científicos que analisaram a associação entre hábito de assistir à TV, consumo alimentar e obesidade em crianças e adolescentes. A identificação dos artigos foi realizada nas bases eletrônicas de dados MedLine, PubMed via Scientific Eletronic Library Online (SciELO-Brasil) e Literatura Latino-Americana e do Caribe em Ciências da Saúde (Lilacs).

A busca foi conduzida no mês de março de 2007. Os unitermos utilizados no MedLine e PubMed foram: television combinado com food intake e obesity. No SciELO-Brasil as palavras-chave utilizadas foram: televisão, consumo alimentar e obesidade, enquanto que no Lilacs utilizou-se a combinação dos unitermos televisão e obesidade.

Estabeleceram-se os seguintes critérios de inclusão: artigos de livre acesso e disponíveis na íntegra; em inglês e português; pesquisas realizadas com ambos os sexos; crianças e adolescentes de 6 a 19 anos de idade; e artigos publicados nos últimos dez anos. Definiu-se não analisar artigos que apresentassem dados referentes a populações adultas ou pré-escolares, artigos não disponíveis na íntegra, notas científicas, comunicações, artigos de revisão, amostras representativas de população indígena e de apenas um dos sexos, amostra contendo sujeitos portadores de outras enfermidades além da obesidade, e artigos de intervenção.

Na busca realizada no MedLine, PubMed encontrou-se um total de 49 artigos. Destes, 19 foram selecionados a partir dos títulos, de acordo com os critérios de inclusão e exclusão estabelecidos. No levantamento realizado no SciELO-Brasil foram encontrados dez artigos, sendo oito selecionados. No Lilacs foram encontrados 16 artigos referentes ao tema, selecionando-se dois, visto que 12 não se enquadravam nos critérios de inclusão, e dois já haviam sido selecionados no SciELO-Brasil.

Ao total, selecionaram-se vinte artigos sobre a associação entre o hábito de assistir à TV com o consumo alimentar e/ou com a obesidade em crianças e adolescentes, sendo que o termo obesidade foi predominantemente usado neste artigo em referência tanto ao diagnóstico de sobrepeso como de obesidade, para fins de padronização de nomenclatura e mais fácil entendimento.

Elaborou-se um roteiro de análise, destacando-se: autoria, país, ano de publicação, delineamento dos estudos, características e tamanho da amostra, principais variáveis desfecho e exposição, principais testes estatísticos e resultados mais importantes.

A partir das variáveis investigadas em cada artigo, os estudos foram agrupados em quatro categorias: a) consumo alimentar em frente à TV e obesidade (três artigos) 12,14,15; b) TV e consumo alimentar (dois artigos) ${ }^{16,17}$; c) TV e obesidade (treze artigos) $)^{9,18-29}$; e d) TV, consumo alimentar e obesidade (dois artigos) ${ }^{7,30}$.

\section{RESULTADOS}

O Anexo sumariza os dados dos vinte artigos selecionados, destacando-se os principais aspectos dos estudos, conforme delineado no roteiro de análise.

Dos artigos analisados, oito foram realizados no Brasil71,15,21-23,25,29,30, seis nos EUA ${ }^{12,16,17,19,20,26}$, 
um em Porto-Rico ${ }^{18}$, um no Canadá24, um na Austrália ${ }^{9}$, um na Suíça ${ }^{14}$, um na China $^{28}$ e um em Dinamarca, Estônia e Portugal ${ }^{27}$. Em relação ao desenho dos estudos, dezessete são de corte transversal 1,9,12,14-16,20-30, dois são longitudinais ${ }^{17,19}$ e um é de caso-controle ${ }^{18}$. A idade dos sujeitos participantes dos estudos variou entre 5 e 19 anos.

\section{Consumo alimentar em frente à TV e obesidade}

Matheson et al. ${ }^{12}$ aplicaram três Recordatórios de 24 horas (R24h), em dias não consecutivos em crianças de $3^{\text {as }}$ séries e adolescentes de $5^{\text {as }}$ séries, residentes na Califórnia. Os autores observaram que o valor energético consumido em frente à TV em dias de final de semana foi cerca de $9 \%$ maior se comparado a dias de semana, e frutas e vegetais foram menos consumidos em frente à TV quando comparados a refeições com a TV desligada. A correlação entre Índice de Massa Corporal (IMC) e densidade energética dos alimentos consumidos em frente à TV não foi significativa para os adolescentes, porém, a correlação entre IMC e percentual de energia proveniente de gorduras dos alimentos consumidos em frente à TV, apesar de fraca, foi significativa nas crianças, em dias de semana.

Stettler et al. ${ }^{14}$ investigaram a associação entre fatores comportamentais e a presença de obesidade em 870 crianças suíças de 6-10 anos. Os autores avaliaram o desfecho obesidade diagnosticado com a presença de sobrepeso pelo critério de Cole et al. ${ }^{31}$, mais a soma das dobras cutâneas subescapular e tricipital igual ou acima do percentil 85, de referência dos EUA. Observou-se que a realização de eventos alimentares (refeições e lanches) em frente à TV não se associou ao estado nutricional. Porém, o tempo diário em frente à TV e o tempo gasto com jogos eletrônicos tiveram associação significativa com a obesidade, em modelo multivariado. Também se observou associação significativa e inversa entre atividade física e obesidade.
Frutuoso et al. ${ }^{15}$ avaliaram o hábito de praticar atividades passivas (assistir à TV, brincar com jogos eletrônicos e usar a Internet) associado ao consumo alimentar e ao sobrepeso e obesidade (definidos pelo critério Cole et al. ${ }^{31}$ ) entre 155 crianças e adolescentes de 7-14 anos, residentes em São Paulo. As autoras encontraram associação significativa entre consumo alimentar em frente à TV e sobrepeso e obesidade em ambos os sexos. Os alimentos consumidos com mais frequência em frente à TV foram: biscoitos, refrigerantes, salgadinhos, pipoca e pães. Praticar atividades passivas teve associação com sobrepeso e obesidade, também em ambos os sexos. Ressalta-se que as análises realizadas nesse estudo foram univariadas e que, em modelos multivariados, possibilita-se mostrar as variáveis independentes mais fortemente associadas a um desfecho, especialmente quando se procede ao controle do modelo para variáveis de confusão. Neste artigo, portanto, não se levou em conta a possível interação de outras variáveis à obesidade, tais como as socioeconômicas, comportamentais e biológicas.

\section{TV e consumo alimentar}

Com o objetivo de avaliar o consumo alimentar de crianças e adolescentes com média de idade de dez anos, Coon et al. ${ }^{16}$ aplicaram três R24h em dias não consecutivos, indagando aos seus pais as atividades realizadas durante as refeições. A quantidade dos alimentos ingeridos foi mensurada por meio de um pôster contendo figuras dos tamanhos das porções, sendo que o primeiro recordatório foi aplicado pessoalmente, e os demais via telefone, pois as crianças receberam os pôsteres. Observou-se associação significativa e inversa entre assistir à TV durante as refeições e nível socioeconômico, escolaridade materna e nível de conhecimento dos pais sobre nutrição. Encontrou-se também associação significativa entre assistir à TV durante as refeições e consumo de carnes vermelhas, pizzas, salgadinhos e refrigerantes, e associação significativa e inversa entre assistir à TV durante as refeições e consumo de frutas e vegetais. 
No estudo de Boynton-Jarret et al. ${ }^{17}$, a energia proveniente do consumo de frutas e verduras apresentou associação inversa com o hábito de assistir à TV, em adolescentes de $10 \mathrm{a}$ 12 anos residentes em Massachuchets (EUA), mesmo após ajuste para variáveis antropométricas, demográficas, consumo percentual de energia proveniente de lipídeos e atividade física.

\section{TV, consumo alimentar e obesidade}

Salmon et al. ${ }^{9}$, avaliando 613 crianças de 5-6 anos e 947 adolescentes de 10-12 anos, matriculados em 24 escolas de Melbourne (Austrália), encontraram associação significativa entre hábito de assistir à TV por um tempo igual ou maior do que duas horas por dia ( $\geq 2 \mathrm{~h} / \mathrm{dia}$ ) e consumo de bebidas altamente energéticas e de petiscos salgados. Observou-se também associação significativa e inversa entre assistir à TV $\geq 2 \mathrm{~h} /$ dia e consumo de frutas. Os autores utilizaram questionário de frequência alimentar aplicado aos pais para avaliar os alimentos consumidos. O questionário continha 83 alimentos e bebidas, além de frutas e vegetais, todos previamente identificados em pesquisa anterior. O hábito de assistir à TV $\geq 2$ horas/dia não apresentou associação significativa com obesidade (definida segundo o critério de Cole et al. ${ }^{31}$ ) quando o modelo de regressão logística foi ajustado por nível socioeconômico, idade, sexo e nível educacional dos pais.

Tanacescu et al. ${ }^{18}$ realizaram estudo de caso-controle com 53 crianças e adolescentes de 7-11 anos, comparando grupo de indivíduos não obesos (IMC/Idade<percentil 85 da população de referência do Centers for Disease Control and Prevention (CDC ${ }^{32}$ ) com grupo de obesos (IMC/Idade $\geq$ percentil 85 do center for disease control ${ }^{32}$ ). Os autores encontraram associação significativa entre obesidade e tempo em frente à TV, associação significativa entre ingestão de doces, salgadinhos, pipoca e amendoins e o hábito de assistir à $T V$, no sexo masculino, e relação entre ver TV e baixa atividade física no sexo feminino.

\section{TV e obesidade}

No estudo de Stettler et al. ${ }^{14}$, com as crianças suíças de 6-10 anos, apesar de os autores não terem observado relação entre consumo alimentar em frente à $T V$, encontraram associação significativa entre hábito de assistir à TV e obesidade, e tempo com jogos eletrônicos e obesidade, em modelo de análise multivariado.

Frutuoso et al. ${ }^{15}$, ao avaliarem as 155 crianças e adolescentes de São Paulo, além de terem encontrado associação significativa entre consumo alimentar em frente à TV e sobrepeso e obesidade em ambos os sexos, observaram que a prática de atividades passivas (assistir à TV, brincar com jogos eletrônicos e usar a Internet) teve associação com sobrepeso e obesidade, também em ambos os sexos.

O principal objetivo de Rose \& Bodor ${ }^{19}$ era avaliar o nível de segurança alimentar e sua relação com a frequência de sobrepeso (percentil

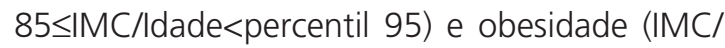
Idade $\geq$ percentil 95 , de acordo com o Centers For Disease Contro/32, em 16889 crianças de 6-7 anos, através de questionário aplicado aos pais. Os autores avaliaram também, de modo prospectivo, a relação entre a segurança alimentar e o ganho de IMC ao longo de um ano. Baseados em 18 questões da escala Household Food Security Scale - Departamento de Agricultura dos EUA - os autores classificaram as moradias em três grupos: a) segurança alimentar, b) insegurança alimentar sem fome e c) insegurança alimentar com fome. Em análise multivariada, os autores também avaliaram a relação da obesidade com outras variáveis, tais como as sócio-demográficas e comportamentais. Assim, foi relatado que $54 \%$ das crianças que sofriam com a insegurança alimentar apresentavam tempo de permanência em frente à $T V>2 h / d i a$. A associação entre assistir à TV $>2 \mathrm{~h} /$ dia e obesidade foi significativa, mas de maneira independente da segurança alimentar.

Mcmurray et al..$^{20}$ avaliaram a influência de atividades físicas e sedentárias, bem como a 
associação da etnia e do nível socioeconômico, no estado de sobrepeso (IMC/Idade $\geq$ percentil 85 conforme National Centers for Health Statistics ${ }^{33}$ ), em 2389 adolescentes de 10-16 anos. Os autores observaram associação significativa e inversa entre nível socioeconômico e hábito de assistir à TV. Assistir à $T V \geq 2 \mathrm{~h} /$ dia e jogar videogame $\geq 2 \mathrm{~h} /$ dia, entretanto, não se associaram significativamente ao sobrepeso, em ambos os sexos, quando foram incluídas, no modelo multivariado, as variáveis etnia e nível socioeconômico.

No estudo de Terres et al. ${ }^{21}$, com adolescentes de 15-18 anos, o tempo gasto com a TV foi categorizado em <2, 3-4, 5-7 e $\geq 8$ horas/dia. Não se encontrou associação univariada, através do teste do qui-quadrado, entre essas categorias e sobrepeso e obesidade definidos pelo critério de Cole et al. ${ }^{31}$. No modelo multivariado, as razões de prevalência não mostraram associação para sobrepeso, em nenhuma das categorias de tempo de audiência à TV. Os adolescentes que relataram estar sob dieta apresentaram prevalência de sobrepeso 1,72 vezes maior, e o fato de omitir refeições também esteve associado. Quanto à obesidade, adolescentes com cinco a oito anos de escolaridade apresentaram prevalência 2,53 vezes maior quando comparados àqueles que possuem segundo grau ou mais. A presença de pais obesos e fazer dieta e omitir refeições também se associaram à obesidade.

No estudo de Oliveira et al..22, com crianças de 5-9 anos, também não foi observada associação significativa entre assistir à TV e obesidade. Vale ressaltar que Oliveira et al..$^{22}$ relataram ter definido sobrepeso e obesidade a partir de IMC igual ou superior aos percentis 85 e 95, respectivamente, adotando os pontos de corte obtidos no estudo de Cole et al..$^{31}$. Destaca-se, entretanto, que percentis e valores de IMC/Idade de Cole et al. ${ }^{31}$ constituem-se em métodos de avaliação de diferentes naturezas classificatórias, não sendo conjugáveis. Os pontos de corte de Cole et al. ${ }^{31}$ foram desenvolvidos com base no método matemático LMS, utilizando-se os pontos de corte de IMC usados para classificação de adultos (25 e
$30 \mathrm{~kg} / \mathrm{m}^{2}$, para sobrepeso e obesidade, respectivamente), enquanto as distribuições percentilares são construídas a partir de conceitos de média e mediana oriundos de dados de alguma amostra estudada. Tais métodos não têm equivalência e, portanto, os valores de IMC/Idade propostos por Cole et al. ${ }^{31}$ não representam os percentis 85 e 95 de quaisquer outros critérios de classificação. Acredita-se, portanto, que o método escolhido por Oliveira et al.22 para avaliar o estado nutricional não tenha sido adequado.

Fonseca et al..$^{23}$, avaliaram 391 adolescentes de 15-17 anos, residentes em Niterói (RJ), investigando a relação de fatores comportamentais e biológicos com o índice de massa corporal. As autoras encontraram associação significativa entre hábito de assistir à TV/jogar videogame/ver vídeos e IMC, no sexo masculino. As variáveis obesidade familiar e fazer dieta para emagrecer, entretanto, foram as mais fortemente associadas ao IMC, em ambos os sexos.

Hanley et al..$^{24}$ avaliaram 242 adolescentes de 10-19 anos, residentes numa comunidade isolada no Canadá. Essa comunidade apresenta alta prevalência de obesidade e diabetes tipo 2 em adultos, por isso, os autores investigaram a prevalência de sobrepeso em adolescentes e os fatores associados à mesma. Observou-se um maior risco de desenvolver sobrepeso (IMC/ldade $\geq$ percentil 85 conforme National Centers for Health Statistics $^{33}$ ) nos adolescentes que assistiam à $T V \geq 5$ h/dia. Para a variável assistir à TV entre 2 e $5 \mathrm{~h} /$ dia não foram encontradas associações significativas.

Dutra et al. ${ }^{25}$ avaliaram a associação do sobrepeso com variáveis demográficas, socioeconômicas, comportamentais e biológicas, em estudo realizado com 810 adolescentes de 10-19 anos residentes em Pelotas (RS). As autoras encontraram que, concomitantemente ao aumento do tempo despendido em frente à TV, ocorreu um incremento da prevalência de sobrepeso (IMC/Idade $\geq$ percentil 85 conforme Must et al. ${ }^{34}$ ). No sexo feminino, assistir à televisão $\geq 4$ horas por dia se associou significativamente ao sobrepeso. 
Eisenmann et al. ${ }^{26}$ observaram resultados similares, sendo que ao aumentar o tempo em frente à TV elevou-se a frequência de sobrepeso (IMC/ldade $\geq$ percentil 85 do Center For Disease Contro $^{32}$ ) e o IMC em adolescentes de 14 a 18 anos, residentes nos EUA. Destaca-se que o estudo desses autores avaliou amostra representativa, abrangendo 15143 adolescentes. Eisenmann et al. ${ }^{26}$ verificaram que ver $\mathrm{TV} \geq 4 \mathrm{~h} / \mathrm{dia}$ (dummy) se associou a sobrepeso, em ambos os sexos. Assim, adolescentes que permanecem de duas a três horas em frente à TV têm 20\% a 25\% menos chance de apresentar sobrepeso, e essa chance aumentou para $40 \%$ nos adolescentes que assistiam uma ou menos horas de TV por dia.

No estudo de Ekelund et al. ${ }^{27}$, o tempo destinado a assistir à TV e à atividade física e sua associação com obesidade (definida pela soma das dobras cutâneas tricipital, subescapular, suprailíaca e bicipital), e medidas bioquímicas foram avaliados em crianças (9-10 anos) e adolescentes (15-16 anos) residentes em três regiões europeias. Os autores não observaram correlação entre tempo dedicado a ver TV e tempo de atividade física, pois o tempo de atividade física foi similar entre os que assistiam pouco $(<0,5 \mathrm{~h} / \mathrm{dia}) \mathrm{e}$ muito ( $>2,5 \mathrm{~h} / \mathrm{dia})$ à televisão. Observou-se associação significativa entre hábito de assistir à TV e obesidade, e TV e insulina de jejum em modelo multivariado ajustado para sexo, idade, maturação sexual, atividade física, peso ao nascer e nível socioeconômico dos pais. Entretanto, ao inserir a atividade física no modelo ajustado, a associação entre ver TV e a insulina de jejum perdeu a significância estatística.

Waller et al..28 estudaram o padrão de atividade física e de consumo alimentar e a relação desses fatores com o sobrepeso (incluindo obesidade), em 1385 crianças e adolescentes chineses de 6-11 anos. O R24h aplicado em três dias foi o instrumento utilizado para avaliar o consumo alimentar, e para investigar questões sobre atividade física e atividades sedentárias os autores utilizaram um questionário sobre os sete dias anteriores à entrevista (semanário). Em relação à TV, observou-se se as crianças e adolescentes avaliados permaneciam pouco tempo em frente à mesma, já que o tempo semanal médio foi de 5,1 horas (eutróficos: Média - M=5,5, Desvio-Padrão - $D P=5,1 \mathrm{~h} / \mathrm{semana}$ e sobrepeso: $M=3,7$, $\mathrm{DP}=4,3 \mathrm{~h} / \mathrm{semana})$. $\mathrm{O}$ fato de permanecer em frente à TV, portanto, não é fator associado ao sobrepeso, já que as crianças eutróficas assistiam significativamente mais à TV do que as com sobrepeso. Vale ressaltar, também, que a prevalência de sobrepeso encontrada foi relativamente baixa se comparada àquela encontrada em outros estudos com crianças e adolescentes, totalizando $9,4 \%$.

O estudo de Silva \& Malina ${ }^{29}$ mostrou que 323 adolescentes de 10-19 anos residentes em Niterói despendiam, em média, 4,7h/dia em frente à $T V$, identificando associação significativa entre o tempo de TV e sobrepeso (IMC/Idade>percentil 85 de acordo com critério de classificação de Himes \& Dietz $\left.^{35}\right)$.

Baruki et al. ${ }^{30}$, em município do Estado de Mato Grosso do Sul, verificaram a associação entre o estado nutricional de 403 crianças de 7-10 anos e a prática de atividades ativas e sedentárias. Os autores constataram correlação fraca e positiva entre tempo despendido em frente à TV e IMC e tempo despendido em frente à TV e percentual de gordura corporal. Os resultados indicaram que as crianças eutróficas passavam menos tempo em frente à TV quando comparadas às com sobrepeso. Resultados semelhantes foram encontrados por Pimenta \& Palma ${ }^{7}$, que avaliaram o hábito de assistir à TV e a prática de atividade física de adolescentes de 10-11 anos. Os autores observaram associação significativa entre hábito de assistir à TV e percentual de gordura corporal, e a média de tempo de assistência à TV foi maior do que o tempo destinado à prática de atividades físicas.

\section{DISCUSS Ã O}

Em relação à influência da TV no consumo alimentar, foi possível observar que seis estudos 
(cinco estrangeiros e um brasileiro), dentre nove (85\% dos artigos), apontam uma interferência negativa da TV. Três estudos identificaram que quanto maior é o tempo dedicado em frente à mesma, menor é o consumo de frutas e vegetais por crianças e adolescentes. Em quatro dos seis estudos observou-se que o consumo de doces, salgadinhos, refrigerantes e alimentos gordurosos é maior quanto maior é o tempo despendido com a TV. Em dois estudos, dentre três que avaliaram o hábito de assistir à televisão durante as refeições, observou-se que se alimentar em frente à TV associou-se à obesidade. É provável que essa interferência seja devida, em boa parte, à publicidade relativa a alimentos de baixo valor nutricional. Por outro lado, a aquisição de alimentos pelas famílias pode ser outro determinante.

No Brasil, dados da Pesquisa de Orçamentos Familiares (POF) 2002-2003 ${ }^{36}$ mostraram que a aquisição de açúcar e refrigerantes pelas famílias brasileiras compreendeu $13,4 \%$ do valor energético, enquanto que o percentual relativo a frutas, verduras e legumes totalizou apenas 2,3\%. Considerando essa realidade, aliada ao fato da TV influenciar o consumo de alimentos ricos em gorduras e açúcares, faz-se necessário regulamentar o marketing sobre alimentos nos canais de TV, já que frutas e hortaliças indubitavelmente não são anunciadas enfaticamente. Chaud \& Marchioni ${ }^{37}$, ao analisarem estudos sobre os alimentos alvo de publicidade, identificaram que é forte o apelo de marketing sobre produtos de saúde e alimentação, e essa ênfase por muitas vezes é realizada sobre características nutricionais que não são exclusivas dos produtos anunciados, mas podem ser encontradas em alimentos in natura.

Vale ressaltar, nos estudos sobre o consumo alimentar, que o QFA e o R24h foram os instrumentos mais utilizados. Ambos os inquéritos dietéticos apresentam limitações, como a possibilidade de ocorrência de erro por sub ou suprarregistro por parte do entrevistado ${ }^{38}$. Segundo a World Health Organization ${ }^{39}$, para se conhecer a ingestão média de forma mais precisa, seria necessário aplicar o R24h de três a quatro vezes, em dias não consecutivos, em função da variabilidade intraindividual da dieta. Apenas três, dentre seis artigos que utilizaram o R24h, aplicaram três vezes esse inquérito, e em dias não consecutivos. Quanto ao QFA, quando aplicado em crianças e adolescentes, deve listar os alimentos que habitualmente são consumidos por estes grupos ${ }^{40}$, assim como fizeram Salmon et al. ${ }^{9}$ em seu estudo com os escolares de Melbourne, na Austrália. Lanches feitos fora de casa e guloseimas como salgadinhos, biscoitos, balas, doces e refrigerantes, também devem ser listados. Dessa maneira, estudos com inquéritos dietéticos devem ser realizados mediante rigoroso planejamento metodológico, incluindo o treinamento da equipe de entrevistadores/coletores de dados para a aplicação dos instrumentos escolhidos, a fim de evitar viés por erro de aferição de dados.

Apesar das limitações metodológicas dos artigos, é importante apontar que as constatações relativas à influência da TV no consumo alimentar de crianças e adolescentes levaram à adoção de distintas iniciativas que visam à promoção da saúde e à prevenção de doenças associadas ao estilo de vida. A American Academy of Pediatrics, por exemplo, sugere que pais ou responsáveis não permitam que suas crianças e adolescentes permaneçam em frente à TV mais que 2 horas/dia ${ }^{41}$. No Brasil, sob a direção da Agência Nacional de Vigilância Sanitária (ANVISA), órgão vinculado ao Ministério da Saúde, foi sancionada a Lei n 11 265, no ano de 2006, que regulamenta a publicidade de alimentos para lactentes e crianças da primeira infância ${ }^{42}$. O objetivo dessa lei é regulamentar a publicidade de fórmulas lácteas, mamadeiras, chupetas e similares, para que não haja desestímulo à amamentação materna. Esse foi o primeiro passo para a que a publicidade não interfira de maneira inadequada nas escolhas alimentares dos indivíduos, sugerindo que outras medidas semelhantes podem ser tomadas quanto ao anúncio de alimentos de baixo valor nutricional.

A associação entre o tempo despendido em frente à televisão e a ocorrência da obesidade 
apareceu em $60 \%$ dos artigos. A maioria dos estudos brasileiros, seis estudos dentre oito, encontrou associação significativa entre horas assistindo à TV e sobrepeso/obesidade, e em um destes, também se verificou associação positiva entre TV e consumo alimentar. Nas investigações estrangeiras, seis dentre dez estudos, apresentaram resultados positivos para a associação entre TV e obesidade ou consumo alimentar em frente à TV e obesidade, além de se identificar associação inversa entre televisão e tempo de atividade física. Quanto à avaliação nutricional, cita-se que a comparação entre os estudos analisados ficou prejudicada devido aos diversos critérios diagnósticos aplicados para a estimativa da prevalência de sobrepeso e/ou obesidade. Sabe-se que até mesmo numa mesma população diferentes critérios podem gerar dados distintos ${ }^{43,44} \mathrm{e}$, por isso, apesar de parecer haver associação entre essas variáveis, vale destacar que um estudo de revisão mais vasto, utilizando artigos com o mesmo critério diagnóstico traria resultados mais consistentes sobre essa associação. Além disso, as diferentes maneiras de se categorizar e analisar a medida de ver TV, pode ter gerado essas discrepâncias.

Outro fator a ser analisado é o fato de a maior parcela dos artigos descritos apresentarem delineamento transversal. Apesar de serem mais rápidos, de menor custo e terem melhor operacionalidade, estudos dessa natureza podem não demonstrar relação de causa e efeito. Para se indicar uma causalidade em estudos epidemiológicos, um conjunto de condições e critérios deve ser atendido pela investigação ${ }^{45}$. Assim, os resultados encontrados em 16 dos vinte estudos analisados podem estar mostrando apenas relações estatísticas de dependência entre variáveis, sem, no entanto, estabelecer o hábito de assistir à TV como causa direta do desenvolvimento do sobrepeso ou da obesidade.

Apesar dessas limitações metodológicas, observou-se também que os estudos analisados apontam associação inversa entre tempo em frente à TV e tempo dedicado à atividade física, o que sugere a substituição das práticas de exercício físico pelo tempo em frente à TV, fatos que, estando presentes no estilo de vida, podem propiciar o sobrepeso e obesidade. Na sociedade contemporânea, parece haver uma tendência a se usar cada vez menos os espaços públicos de lazer, o que pode ser devido ao problema da violência, principalmente em grandes aglomerados urbanos. Em uma amostra de pré-escolares, investigada em uma cidade dos EUA, observou-se associação significativa e positiva entre a percepção de mães a respeito da segurança na vizinhança e o tempo que seus filhos despendiam em frente à $T V$, mostrando que se permanecia mais em frente à TV quando o nível de segurança não permitia que se brincasse fora de casa ${ }^{46}$.

\section{CONSIDERAÇÕES FINAIS}

Por fim, salienta-se que os resultados dos estudos apontam a permanência em frente à TV como fator que influencia crianças e adolescentes a desenvolverem hábitos alimentares menos saudáveis, e também reduz o tempo dedicado à atividade física.

Apesar de não haver concordância entre os estudos internacionais a respeito da influência da TV na obesidade, destaca-se que as mudanças comportamentais provocadas por ela no hábito alimentar e no tempo dedicado à atividade física são determinantes que podem propiciar o sobrepeso ou a obesidade, indicando a TV como variável que pode estar indiretamente associada. Por isso, em estudos epidemiológicos nutricionais, é de grande valia analisar o tempo dedicado a assistir à TV, em crianças e adolescentes.

Esses achados devem alertar as autoridades públicas, para que programas e políticas continuem enfatizando a promoção da alimentação saudável e a prevenção da obesidade nas mais tenras idades, podendo incluir medidas que estimulem o lazer ativo e a redução do tempo que crianças e adolescentes permanecem em frente à TV, bem como a regulamentação da publicidade de alimentos, tais como aquelas já adotadas por órgãos de saúde nos EUA e no Brasil. 
616 | C.E. ROSSI et al.

\section{COLABORADORES}

C.E. ROSSI e D.E. ALBERNAZ participaram da revisão da literatura e redação do artigo. F.A.G. VASCONCELOS orientou o desenho metodológico da investigação e revisou o artigo. P.F. DI PIETRO e M.A.A. DE ASSIS participaram da discussão e revisão do artigo.

\section{REFERÊ N CIAS}

1. World Health Organization. Nutrition for health and development: a global agenda for combating malnutrition. Geneva: WHO; 2003 [cited 2007 May 15]. Available from: <http://www.who.int/ mip2001/files/2231/NHDprogressreport2000.pdf>.

2. World Health Organization. The challenge of obesity in the WHO European Region and the strategies for response. Dinmark: WHO; 2007.

3. Wang Y, Monteiro C, Popkin BM. Trends of obesity and underweight in older children and adolescents in the United States, Brazil, China, and Russia. Am J Clin Nutr. 2002; 75(6)971-7.

4. Kain J, Andrade M. Characteristics of the diet and patterns of physical activity in obese Chilean preschoolers. Nutr Res. 1999; 19(2):203-15.

5. Monteiro CA, Mondini L, Costa RBL. Mudanças na composição e adequação nutricional da dieta familiar nas áreas metropolitanas do Brasil (2488-2496). Rev Saúde Pública. 2000; 34(3):251-8.

6. Kiess W, Galler A, Reich A, Müller G, Kapellen T, Deutscher J, et al. Clinical aspects of obesity in childhood and adolescence. Obes Rev. 2001; 21(2): 29-36.

7. Pimenta APAA, Palma A. Perfil epidemiológico da obesidade em crianças: relação entre televisão, atividade física e obesidade. Rev Bras Cien Mov. 2001; 9(4):19-24.

8. Assis MAA, Rolland-Cachera MF, Vasconcelos FAG, Bellisle F, Calvo MCM, Luna MEP, et al. Overweight and thinness in 7-9 year old children from Florianópolis, Southern, Brazil: a comparison with a French study using a similar protocol. Rev Nutr. 2006; 19(3):299-308. doi: 10.1590/S1415-5273 2006000300001.

9. Salmon J, Campbell KJ, Crawford DA. Television viewing habits associated with obesity risk factors: a survey of Melbourne schoolchildren. MJA. 2006; 184(2):64-7.

10. Almeida SS, Nascimento PCBD, Quaioti TCB. Quantidade e qualidade de produtos alimentícios anunciados na televisão brasileira. Rev Saúde Pública. 2002; 36(3):353-5.
11. Story M, Faulkner P. The Prime Time Diet: A content analysis of eating behavior and food messages in television program content and commercials. Am J Public Health. 1990; 80(6):736-40.

12. Matheson DM, Killen JD, Wang Y, Varady A, Robinson TN. Children's food consumption during television viewing. Am J Clin Nutr. 2004; 79(1): 1088-94.

13. Fiates GMR, Amboni RDMC, Teixeira E. Television use and food choices of children: qualitative approach. Appetite. 2007; 50:12-8.

14. Stettler N, Singer TM, Suter PM. Electronic games and environmental factors associated with childhood obesity in Switzerland. Obes Res. 2004; 12(6):896-903.

15. Frutuoso MFP, Bismarck-Nasr EM, Gambardella AMD. Redução do dispêndio energético e excesso de peso corporal em adolescentes. Rev Nutr. 2003; 16(3):257-63. doi: 10.1590/S1415-52732003000 300003.

16. Coon KA, Goldberg J, Rogers BL, Tucker KL. Relationships between use of television during meals and children's food consumption patterns. Pediatrics. 2001; 107(1):e7.

17. Boynton-Jarrett R, Thomas TN, Peterson KE, Wiecha J, Sobol AM, Gortmaker SL. Impact of television viewing patterns on fruit and vegetable consumption among adolescents. Pediatrics. 2003; 112(6):1321-6.

18. Tanacescu M, Ferris AM, Himmelgreen DA, Rodriguez N, Pérez-Escamilla R. Biobehavioral factors are associated with obesity in Puerto Rican children. J Nutr. 2000; (130):1734-42.

19. Rose D, Bodor N. Household food insecurity and overweight status in young school children: results from the early childhood longitudinal study. Pediatrics. 2006; 117(2):464-73.

20. Mcmurray RG, Harrel JS, Deng S, Bradley CB, Cox LM, Bangdiwala SI. The influence of physical activity, socioeconomic status, and ethnicity on the weight status of adolescents. Obes Res. 2000; 8(2): 240-8.

21. Terres NG, Pinheiro RT, Horta BL, Pinheiro KAT, Horta LL. Prevalência e fatores associados ao sobrepeso e à obesidade em adolescentes. Rev Saúde Pública. 2006; 40(4):627-33.

22. Oliveira AMA, Cerqueira EMM, Souza JS, Oliveira AC. Sobrepeso e obesidade infantil: influência de fatores biológicos e ambientais em Feira de Santana, BA. Arq Bras Endocrinol Metab. 2003; 47(2): 144-50.

23. Fonseca VM, Sichieri R, Veiga GV. Fatores associados à obesidade em adolescentes. Rev Saúde Pública. 1998; 32(6):541-9. 
24. Hanley AJG, Harris SB, Gittelsohn J, Wolever TMS, Saksvig B, Zinman B. Overweight among children and adolescents in a Native Canadian community: prevalence and associated factors. Am J Clin Nutr. 2000; 71:693-700.

25. Dutra $C L$, Araújo $C L$, Bertoldi $A D$. Prevalência de sobrepeso em adolescentes: um estudo de base populacional em uma cidade no sul do Brasil. Cad Saúde Pública. 2006; 22(1):151-62.

26. Eisenmann JC, Todd BR, Wang MQ. Physical activity, TV viewing, and weight in U.S. youth: 2499 Youth Risk Behavior Survey. Obes Res. 2002; 10(5):379-85.

27. Ekelund U, Brage S, Froberg K, Harro M, Anderssen SA, Sardinha LB, et al. TV viewing and physical activity are independently associated with metabolic risk in children: The European Youth Study. PLos Medicine. 2006; 3(12):1949-56.

28. Waller CE, DU S, Popkin BM. Patterns of overweight, inactivity, and snacking in chinese children. Obes Res. 2003; 11:957-61.

29. Silva RCR, Malina RM. Sobrepeso, atividade física e tempo de televisão entre adolescentes de Niterói, Rio de Janeiro, Brasil. Rev Bras Cien Mov. 2003; 11(4):63-6.

30. Baruki, SBC, Rosado LEPL, Rosado GP, Ribeiro RCL. Associação entre estado nutricional e atividade física em escolares da Rede Municipal de Ensino em Corumbá - MS. Rev Bras Med Esporte. 2006; 12 (2):90-94.

31. Cole TJ, Bellizzi MC, Flegal KM, Dietz WH. Establishing a standard definition for child overweight and obesity worldwide: international survey. BMJ. 2000; 320:1240-3.

32. Centers for Disease Control and Prevention CDC Growth Charts: United States; 2000. Number 314. [cited 2007 May 15]. Available from: <http://www. cdc.gov/nchs>.

33. National Center for Health Statistics. Anthropometric Reference Data and prevalence of overweight: United States 1976-80. Washington (DC): Public Health Service; 1987 Vital and Health Statistics: series 11: 238. DHHS publication PHS 87-1688.

34. Must A, Dallal GE, Dietz WH. Reference data for obesity: $85^{\text {th }}$ and $95^{\text {th }}$ percentiles of body mass index $\left(w t / h t^{2}\right)$ and triceps skinfold thickness. Am J Clin Nutr. 1991; 53(4):839-46.

35. Himes J, Dietz W. Guidelines for overweight in adolescent preventive services: recommendations from an expert committee. Am J Clin Nutr. 1994; 59:307-16.
36. Brasil. Pesquisa de Orçamentos Familiares 2002-2003: análise da disponibilidade domiciliar de alimentos e do estado nutricional no Brasil. Rio de Janeiro: IBGE; 2004.

37. Chaud DMA, Marchioni DML. Nutrição e mídia: uma combinação às vezes indigesta. Hig Aliment. 2004; 18(116-117):18-22.

38. Vasconcelos FAG. Tendências históricas dos estudos dietéticos no Brasil. Hist Ciênc Saúde - Manguinhos. 2007; 14(1):197-219.

39. World Health Organization. Preparation and use of food-based dietary guidelines. Geneva: WHO; 1998. Technical Report Series, 880.

40. Falcão-Gomes RC, Coelho AAS, Schimitz BAS. Caracterização dos estudos de avaliação do consumo alimentar de pré-escolares. Rev Nutr. 2006; 19(6): 713-27. doi: 10.1590/\$1415-52732006000600 008.

41. American Academy of Pediatrics, Committee on Nutrition. Policy statement: prevention of pediatric overweight and obesity. Pediatrics. 2003; 112(2):424-30.

42. Brasil. Presidência da República, Casa Civil. Lei $n^{\circ}$ 11.265, de 3 de janeiro de 2006. Regulamenta a comercialização de alimentos para lactentes e crianças de primeira infância e produtos de puericultura correlatos. Diário Oficial da União. 2006; 4 jan.; Seção 1, p.1.

43. Assis MAA, Rolland-Cachera MF, Grosseman S, Vasconcelos FAG, Luna MEP, Calvo MCM, et al. Obesity, overweight and thinness in schoolchildren of the city of Florianopolis, Southern Brazil. Eur J Clin Nutr. 2005; 59:1015-21.

44. Rolland-Cachera MF, Castelbon K, Arnault N, Bellisle F, Romano RC, Lehingue $Y$, et al. Body mass index in 7-9-y-old French children: frequency of obesity, overweight and thinness. Inter J Obes. 2002; 26:1610-6.

45. Peres MA, Antunes JLF. O método epidemiológico de investigação e sua contribuição para a saúde bucal. In: Fundamentos de odontologia: epidemiologia da saúde bucal. Rio de Janeiro: Guanabara Koogan; 2006. Capítulo 1, p.3-24.

46. Burdette HL, Whitaker RC. A National study of neighborhood safety, outdoor play, television viewing and obesity in preschool children. Pediatrics. 2005; 116(3):657-62.

Recebido em: 17/4/2008

Versão final reapresentada em: 12/11/2009 Aprovado em: 17/3/2010 
ANEXO

RELAÇÃO DOS ESTUDOS SOBRE HÁBITO DE ASSISTIR À TELEVISÃO, CONSUMO ALIMENTAR E OBESIDADE EM CRIANÇAS E ADOLESCENTES, PUBLICADOS NO PERÍODO DE 1998 A 2006, EM ORDEM CRONOLÓGICA E DE ACORDO COM: AUTORIAVANO DE PUBLICAÇÃO E LOCAL DE REALIZAÇÃO, DESENHO DO ESTUDO, FAIXA ETÁRIA E TAMANHO DA AMOSTRA, VARIÁVEIS DESFECHO E PRINCIPAIS VARIÁVEIS EXPOSIÇÃO, RESULTADOS PRINCIPAIS E ANÁLISE ESTATÍSTICA

\begin{tabular}{|c|c|c|c|c|}
\hline $\begin{array}{l}\text { Autoria/Ano de } \\
\text { publicação/local }\end{array}$ & Desenho & Amostra & Desfecho e exposição & $\begin{array}{l}\text { Resultados principais e } \\
\text { análise estatística }\end{array}$ \\
\hline \multirow[t]{2}{*}{$\begin{array}{l}\text { Baruki et al. }{ }^{30} \\
2006 \text { Brasil }\end{array}$} & \multirow[t]{2}{*}{ Transversal } & \multirow[t]{2}{*}{$\begin{array}{l}403 \text { crianças } \\
\text { (7-10 anos) }\end{array}$} & $\begin{array}{l}\text { Desfecho: risco de sobrepeso se } \\
\text { IMC/Idade } \geq \text { percentil } 85 \text { e }<95 \\
\text { e sobrepeso se IMC/Idade } \\
\text { zpercentil } 95 \text { do CDC } 32\end{array}$ & \multirow{2}{*}{$\begin{array}{l}\text { Crianças com sobrepeso perma- } \\
\text { neciam mais tempo na TV e nos } \\
\text { jogos eletrônicos se comparadas } \\
\text { às eutróficas e com risco de } \\
\text { sobrepeso }(p<0,05) \text {. Teste de } \\
\text { Tukey }\end{array}$} \\
\hline & & & $\begin{array}{l}\text { Exposição: tempo de TV e de } \\
\text { jogos eletrônicos/dia }\end{array}$ & \\
\hline
\end{tabular}

Dutra et al..$^{25}$

2006 Brasil

Ekelund et al. ${ }^{27} 2006$ Dinamarca, Estônia e Portugal

Rose \& Bodor ${ }^{19} 2006$
Estados Unidos da
América

Salmon et al. ${ }^{9}$ 2006 Austrália

Terres et al..$^{21}$ 2006 Brasil
Transversal, de

base populacional

Principal análise: Longitudinal; Para a análise do desfecho com o tempo de televisão: Transversal aninhado em longitudinal

Transversal

2460 escolares (5-6 anos e 10-12 anos)

Transversal, de

base populacional
960 adolescentes (15-18 anos)
10 adolescentes (10-19 anos)

16889 crianças (6-7 anos)

1092 crianças (9-10 anos) e (15-16 anos)
Desfecho: sobrepeso e obesidade conforme Cole et al. ${ }^{31}$ Exposição: Consumo alimentar energéticas (OR=2,31; $95 \%$ e tempo de TV

\section{Id}

Exposição: variáveis demográficas, socioeconômicas, comportamentais e biológicas, dentre elas o tempo de TV/dia

\section{Três modelos:}

a) TV e adiposidade como desfechos e medidas bioquímicas e 0,$11 ; p<0,05)$ e TV e insulina de adiposidade como exposições; jejum $(\beta=0,067 ; 95 \%$ IC $=0,014$ b) medidas bioquímicas como 0,$12 ; p<0,05)$. desfechos e ver TV como expo- Associação não significativa ensição; tre TV e medidas bioquímicas c) análise post hoc: comer assis- $(\beta=0,026 ; \beta=0,053)$./Regressão tindo à TV na associação entre linear multivariada. TV e adiposidade

Desfecho: sobrepeso se IMC/ Associação entre insegurança Idade de do $C D C^{32} \geq$ percentil alimentar e ver $\mathrm{TV}>2 \mathrm{~h} / \mathrm{dia}$ 85 e $<95$ e obesidade se IMC/ $(p<0,05)$. Qui-quadrado. Idade $\geq$ percentil $95 \quad$ Associação entre ver TV $>2 \mathrm{~h} /$ dia Exposição:Segurança alimentar e obesidade (OR=1,24; $95 \%$ e varáveis comportamentais, IC=1,12-1,38; $p<0,001)$./Recomo $>2 \mathrm{~h} /$ dia de TV gressão logística multivariada. $\mathrm{IC}=1,61-3,32)$ e de salgadinhos $\geq 1$ porção/dia (OR=1,50; 95\% $\mathrm{IC}=1,04-2,24)$.TV $\geq 2 \mathrm{~h} /$ dia e obesidade não se associaram (OR=1,37; 95\% IC=0,94-2,00)./ Regressão logística multivariada.

Desfecho: sobrepeso e obesida- Fazer dieta ( $R P=1,72$; IC 95\%: de conforme Cole at al. $\left.{ }^{31} 1,24-2,38\right)$, e omitir refeições Exposição: variáveis socioeco- associaram-se a sobrepeso nômicas, comportamentais e ( $R P=1,69$; IC 95\%: 1,29-2,21). demográficas, dentre elas o tem- TV não se associou a sobrepepo de TV em horas/dia (3-4h, so e obesidade./Qui-quadrado e $5-7 \mathrm{~h}$ ou $>8 \mathrm{~h} / \mathrm{dia}$ ) Regressão de Poisson multivariada. 


\section{ANEXO}

RELAÇÃO DOS ESTUDOS SOBRE HÁBITO DE ASSISTIR À TELEVISÃO, CONSUMO ALIMENTAR E OBESIDADE EM CRIANÇAS E ADOLESCENTES, PUBLICADOS NO PERÍODO DE 1998 A 2006, EM ORDEM CRONOLÓGICA E DE ACORDO COM: AUTORIAVANO DE PUBLICAÇÃO E LOCAL DE REALIZAÇÃO, DESENHO DO ESTUDO, FAIXA ETÁRIA E TAMANHO DA AMOSTRA, VARIÁVEIS DESFECHO E PRINCIPAIS VARIÁVEIS EXPOSIÇÃO, RESULTADOS PRINCIPAIS E ANÁLISE ESTATÍSTICA

\begin{tabular}{|c|c|c|c|c|}
\hline $\begin{array}{l}\text { Autoria/Ano de } \\
\text { publicação/local }\end{array}$ & Desenho & Amostra & Desfecho e exposição & $\begin{array}{c}\text { Resultados principais e } \\
\text { análise estatística }\end{array}$ \\
\hline $\begin{array}{l}\text { Matheson et al. }{ }^{12} \\
2004 \text { Estados Unidos } \\
\text { da América }\end{array}$ & $\begin{array}{l}\text { Transversal, com } \\
\text { duas diferentes } \\
\text { amostras }\end{array}$ & $\begin{array}{l}1^{\mathrm{a}} \text { amostra: } 91 \\
\text { crianças de } 3^{\mathrm{a}} \text { série } \\
2^{\mathrm{a}} \text { amostra: } 124 \text { ado- } \\
\text { lescentes de } 5^{\mathrm{a}} \text { série } \\
\text { (7-12 anos) }\end{array}$ & $\begin{array}{l}\text { Desfecho: IMC } \\
\text { Exposição: Percentual de ener- } \\
\text { gia proveniente de gordura in- } \\
\text { gerida em frente à TV, tipos de } \\
\text { alimentos consumidos em fren- } \\
\text { te à TV }\end{array}$ & $\begin{array}{l}\text { Correlação fraca entre IMC e } \\
\text { consumo de gorduras em fren- } \\
\text { te à } T V \text {, em dias de semana } \\
\text { ( } r=0,25 \text { e } p<0,04 \text { )./Correlação } \\
\text { de Sperman, Análise de variân- } \\
\text { cia multinível. }\end{array}$ \\
\hline $\begin{array}{l}\text { Stettler et al. }{ }^{14} \\
2004 \text { Suiça }\end{array}$ & Transversal & $\begin{array}{l}870 \text { crianças } \\
\text { (6-10 anos) }\end{array}$ & $\begin{array}{l}\text { Desfecho: obesidade se há } \\
\text { sobrepeso por Cole et al. }{ }^{31} \text { e } \\
\text { soma das dobras cutâneas trici- } \\
\text { pital e subescapular } \geq \text { percentil } \\
85 \\
\text { Exposição: tempo diário em } \\
\text { frente à TV, tempo diário em jo- } \\
\text { gos eletrônicos, e assistir à TV } \\
\text { durante as refeições e lanches } \\
\text { em dias de semana }\end{array}$ & $\begin{array}{l}\text { Consumo em frente à TV e so- } \\
\text { brepeso não se associaram. As- } \\
\text { sociação entre TV e sobre-peso } \\
\text { (OR=2,83; } 95 \% \text { IC }=2,08-3,86) \text {, } \\
\text { e jogos eletrônicos e obesida- } \\
\text { de }(O R=2,03 ; \text { IC } 95 \%=1,57- \\
2,61 ; p<0,001) \text {. Associação in- } \\
\text { versa entre atividade física e } \\
\text { obesidade (OR=0,80; IC } \\
95 \%=0,72-0,88 ; p<0,001) \text {. /Re- } \\
\text { gressão logística multivariada. }\end{array}$ \\
\hline $\begin{array}{l}\text { Boynton-Jarrett } \\
\text { et al. }{ }^{17} 2003 \text { Estados } \\
\text { Unidos da América }\end{array}$ & $\begin{array}{l}\text { Longitudinal } \\
\text { prospectivo } \\
\text { Baseline em } 1995 \\
\text { e follow-up em } \\
1997\end{array}$ & $\begin{array}{l}548 \text { adolescentes } \\
\text { (10-12 anos) }\end{array}$ & $\begin{array}{l}\text { Desfecho: consumo de frutas e } \\
\text { verduras } \\
\text { Exposição: consumo de frutas } \\
\text { e verduras no baseline, tempo de } \\
\text { TV e mudanças no tempo em } \\
\text { frente à TV no período de dois } \\
\text { anos }\end{array}$ & $\begin{array}{l}\text { Associação inversa entre con- } \\
\text { sumo diário de frutas e verdu- } \\
\text { ras e TV (baseline: } \beta=-0,16 \text {; } \\
95 \% I C=0,22-0,10 ; p<0,008 \text {; } \\
\text { follow-up: } \beta=-0,16 ; 95 \% \\
\text { IC=0,22-0,07; } p<0,025 \text { )./Re- } \\
\text { gressão linear multivariada. }\end{array}$ \\
\hline $\begin{array}{l}\text { Frutuoso et al. }{ }^{15} \\
2003 \text { Brasil }\end{array}$ & Transversal & $\begin{array}{l}155 \text { crianças e ado- } \\
\text { lescentes } \\
\text { (7-14 anos) }\end{array}$ & $\begin{array}{l}\text { Desfecho: sobrepeso e obesida- } \\
\text { de conforme Cole at al. }{ }^{31} \\
\text { Exposição: Consumo alimentar } \\
\text { em frente à TV, e prática de ati- } \\
\text { vidades passivas: ver TV, brincar } \\
\text { com jogos eletrônicos e perma- } \\
\text { necer na Internet }\end{array}$ & $\begin{array}{l}\text { Associação entre sobrepeso e } \\
\text { obesidade e consumo alimen- } \\
\text { tar em frente à TV para meni- } \\
\text { nos e meninas ( } p<0,001) \text {. Asso- } \\
\text { ciação entre atividades passivas } \\
\text { e sobrepeso e obesidade (pa- } \\
\text { ra os meninos se } 14 \text { a } 28 \mathrm{~h} / \mathrm{se}- \\
\text { mana; } p=0,04 \text {; e para as me- } \\
\text { ninas se }+28 \mathrm{~h} / \mathrm{sem} \text { ana; } \\
p=0,02) \text {./Qui-quadrado e } t \text { de } \\
\text { Student. }\end{array}$ \\
\hline $\begin{array}{l}\text { Oliveira et al. }{ }^{22} \\
2003 \text { Brasil }\end{array}$ & Transversal & $\begin{array}{l}699 \text { criança } \\
\text { (5-9 anos) }\end{array}$ & $\begin{array}{l}\text { Desfecho: sobrepeso se IMC/ } \\
\text { Idade } \\
\text { zpercentil } 85 \text { e obesidade se } \\
\text { IMC/Idade } \geq \text { percentil } 95 \text {, adotan- } \\
\text { do-se os pontos de corte de Cole } \\
\text { et al. }{ }^{31} \\
\text { Exposição: Tempo de TV, de } \\
\text { videogame e de computador }\end{array}$ & $\begin{array}{l}\text { Ver TV, usar computador e jo- } \\
\text { gar videogame não se asso- } \\
\text { ciaram à obesidade ( } p=0,17 \text {; } \\
0,10 ; 0,84 \text {, respectivamente)./ } \\
\text { Regressão logística multiva- } \\
\text { riada. } \\
\text { As razões de chance e Interva- } \\
\text { los de confiança não foram } \\
\text { mostrados. }\end{array}$ \\
\hline $\begin{array}{l}\text { Silva \& Malina } 29 \\
2003 \text { Brasil }\end{array}$ & Transversal & $\begin{array}{l}323 \text { adolescentes } \\
\text { (10-19 anos) }\end{array}$ & $\begin{array}{l}\text { Desfecho: sobrepeso se IMC/ } \\
\text { Idade>percentil } 85 \text { de Himes \& } \\
\text { Dietz }^{35}\end{array}$ & $\begin{array}{l}\text { Associação entre TV }>3 \text { horas e } \\
\text { sobrepeso (OR=1,17; } p<0,05) \text {./ } \\
\text { Regressão logística multivariada. }\end{array}$ \\
\hline $\begin{array}{l}\text { Waller et al. }{ }^{28} \\
2003 \text { China }\end{array}$ & $\begin{array}{l}\text { Transversal } \\
\text { aninhado em } \\
\text { longitudinal }\end{array}$ & $\begin{array}{l}1385 \text { crianças e } \\
\text { adolescentes } \\
\text { (6-11 anos) }\end{array}$ & $\begin{array}{l}\text { Exposição: tempo de TV/dia } \\
\text { Desfecho: sobrepeso se IMC/ } \\
\text { Idade>percentil } 85 \text { do CDC } 32 \\
\text { Exposição: Atividades sedentá- } \\
\text { rias, como tempo de TV e de jo- } \\
\text { gos eletrônicos em horas/sema- } \\
\text { na }\end{array}$ & $\begin{array}{l}\text { A exposição não teve relação } \\
\text { com o desfecho, pois os eutró- } \\
\text { ficos passavam somente cerca } \\
\text { de } 15 \text { minutos/dia a mais assis- } \\
\text { tindo à TV do que os com } \\
\text { sobrepeso }(p<0,02) \text {. /Teste } t \text { de } \\
\text { Student. }\end{array}$ \\
\hline
\end{tabular}


ANEXO

RELAÇÃO DOS ESTUDOS SOBRE HÁBITO DE ASSISTIR À TELEVISÃO, CONSUMO ALIMENTAR E OBESIDADE EM CRIANÇAS E ADOLESCENTES, PUBLICADOS NO PERÍODO DE 1998 A 2006, EM ORDEM CRONOLÓGICA E DE ACORDO COM: AUTORIAJANO DE PUBLICAÇÃO E LOCAL DE REALIZAÇÃO, DESENHO DO ESTUDO, FAIXA ETÁRIA E TAMANHO DA AMOSTRA, VARIÁVEIS DESFECHO E PRINCIPAIS VARIÁVEIS EXPOSIÇÃO, RESULTADOS PRINCIPAIS E ANÁLISE ESTATÍSTICA

Conclusão

\begin{tabular}{|c|c|c|}
\hline $\begin{array}{l}\text { Autoria/Ano de } \\
\text { publicação/local }\end{array}$ & Desenho & Amostra \\
\hline $\begin{array}{l}\text { Eisenmamm et al. }{ }^{26} \\
2002 \text { Estados Unidos } \\
\text { da América }\end{array}$ & Transversal & $\begin{array}{l}15143 \text { adolescen- } \\
\text { tes } \\
\text { (14-18 anos) }\end{array}$ \\
\hline
\end{tabular}

Coon et al. ${ }^{16}$ 2001 Estados Unidos da América

Pimenta \& Palma7 2001 Brasil

Hanley et al. ${ }^{24}$ 2000 Canadá

Mcmurray et al. ${ }^{20}$ 2000 Estados Unidos da América

Tanacescu et al. ${ }^{18}$ 2000 Porto Rico

Fonseca et al. ${ }^{23}$ 1998 Brasil
Transversal

(setembro de

1993 a junho de

91 crianças (idade média dez anos) 1995)

Transversal

56 crianças e adolescentes (10-11 anos)

Transversal

242 adolescentes (10-19 anos)
Transversal aninhado em longitudinal

Caso-controle

53 crianças e adolescentes (7-11 anos) 31 meninas, 19 obesas e 12 controles, e 22 meninos, dez obesose 12 controles

Transversal

391 adolescentes (15-17 anos)
Desfecho e exposição

Resultados principais e análise estatística

Desfecho: sobrepeso se IMC/ IMC e tempo de TV foram direIdade $\geq$ percentil 85 do $C C^{32}$ tamente proporcionais, em amExposição: tempo de TV em dia bos os sexos $(p<0,01)$./Análise escolar e tempo de atividade fí- de covariância. sica

Ver $\mathrm{TV} \geq 4 \mathrm{~h} /$ dia se associou a sobrepeso, em ambos os sexos ( O" $2-3 \mathrm{~h} / \mathrm{dia}$ OR=0,78; $95 \%$ $\mathrm{IC}=0,61-0,99 ; p=0,04$; e $\mathrm{O}$ $\mathrm{OR}=0,74 ; 95 \% \mathrm{IC}=0,54-1,01$; $\mathrm{p}=0,0001)$./Regressão logística multivariada.

Desfecho: comer enquanto se Associação entre o desfecho e assiste à TV consumo de carnes vermelhas Exposição: frequência do con- $(\beta=0,14 ; p<0,01)$, pizzas e salsumo de alguns alimentos e gados $(\beta=0,12 ; p<0,05)$, e repercentual de energia proveni- frigerantes $(\beta=0,15 ; p<0,05)$; e ente de alguns alimentos inversa com o consumo de fru$\operatorname{tas}(\beta=-0,01, p \leq 0,05)$ e vegetais $(\beta=-0,01, p \leq 0,01)$./Regressão linear multivariada.

Desfecho:percentual de gordu- Associação entre TV e percenra pelas fórmulas de Slaughter tual de gordura corporal et al. (1988) e Lohman (1987) $\quad(r=0,51$ e $p<0,01)$./Teste $t$ de Exposição: tempo de TV Student.

Desfecho: sobrepeso se IMC/ TV $\geq 5 \mathrm{~h} /$ dia se associou a soIdade $\geq$ percentil 85 do NANHES brepeso $(p=0,03 ; \mathrm{OR}=2,52$; III (1998) $95 \% \mathrm{IC}=1,06-5,98) . /$ Regressão Exposição: TV $<2 \mathrm{~h}, 3-4$ h e $\geq$ logística multivariada. 5h/dia.

Associação não significativa entre TV e sobrepeso (meninos: OR $=1,07 ;$ IC $95 \%=0,73-1,55$; meninas: OR 1,424; IC 95\% =

2389 adolescentes Desfecho: sobrepeso se IMC/ 0,969-2,091)./Associação não Idade $\geq$ percentil 85 do NCHS $^{33}$ significativa entre jogar Exposição: ver TV>2 h/dia e jo- videogame e sobrepeso (menigar videogame $>2 \mathrm{~h} / \mathrm{dia}$, etnia e nos: OR =1,08; IC 95\%=0,76nível educacional dos pais 1,51; meninas: $\mathrm{OR}=0,68$; IC $95 \%=0,415-1,17) . /$ Regressão logística multivariada.

Desfecho: obesidade se IMC/ Associação entre ingerir doces, Idade $\geq$ percentil 85 das referên- salgadinhos, pipoca e amencias do NANHES doins e ver TV em dias de seExposição: variáveis biológicas, mana $(r=0,57 ; p=0,007)$ e final socioeconômicas e comporta- de semana $(r=0,60 ; p=0,003)$, mentais, entre elas: consumo em nos meninos. Relação inversa frente à TV, horas/dia de TV, entre TV e atividade física no computador e videogames sexo feminino $(p<0,05)$./Correlação de Spearman.

Desfecho: IMC como variável Associação entre obesidade e contínua TV $(O R=1,86 ; 95 \%$ IC $=1,02$ Exposição: variáveis comporta- 3,$42 ; p=0,04)$./Regressão logísmentais e biológicas, dentre elas tica multivariada. o tempo de TV/videogamel As vídeos por dia

Associação entre TV/videogame/ vídeos e IMC $\left(\beta=0,27 ; R^{2}=0,24\right.$ e $p=0,02)$, no sexo masculino./ Regressão linear multivariada. 